\title{
Avaliação da pectina-HPMC no processo de revestimento por compressão. I - Estudo da propriedade de intumescimento em núcleos revestidos
}

\author{
Gabriela Gomes Guimarães, Gleyckson Itsuo Katsuki, Naira Denise Zanardo, Douglas Abramoski \\ Ribeiro, Osvaldo Albuquerque Cavalcanti*
}

Laboratório de Tecnologia Farmacêutica, Departamento de Farmácia e Farmacologia, Universidade Estadual de Maringá

*Correspondência:

O. A. Cavalcanti

Laboratório de Tecnologia

Farmacêutica

Departamento de Farmácia e

Farmacologia

Universidade Estadual de Maringá

Av. Colombo, 5790, Anexo Bloco P02

87020-900 - Maringá - PR, Brasil

E-mail: oacavalcanti@uem.br
Neste estudo, comprimidos de teofilina anidra foram revestidos através do processo de revestimento por compressão aplicando sistema binário pectina-HPMC. O método aplicado gerou comprimidos revestidos com características fisicas adequadas aos padrões farmacopeicos. Foram avaliadas as características físicas dos comprimidos obtidos em diferentes proporções (80:20, 60:40, 50:50, 40:60, 20:80, 0:100) de pectina-HPMC respectivamente, e estabelecido o perfil de intumescimento desses sistemas nos fluidos de simulação gástrico $(p H 1,2)$ e intestinal $(p H$ 6,8). $O$ método aplicado gerou a formação de comprimidos revestidos que apresentaram variação nos perfis de hidratação in vitro, entretanto, a análise estatística revelou que estas diferenças não foram significativas quando comparadas entre si. O sistema formado apresenta elevada expectativa sobre o gerenciamento da liberação de fármacos, todavia, só a partir do teste de dissolução que constitui a segunda etapa deste projeto poderemos definir qual das formulações propostas será mais eficaz no controle da cinética de liberação.

\author{
Unitermos \\ - Comprimidos/revestimento \\ por compressão \\ - Comprimidos/avaliação \\ - Teofilina \\ - Pectina \\ - Fármacos/liberação
}

\section{INTRODUÇÃO}

O emprego das formas farmacêuticas sólidas orais convencionais destinadas ao tratamento de algumas patologias depara com uma série de exigências fisiológicas inerentes ao trato gastrintestinal (TGI). Estas exigências podem ser consideradas verdadeiros obstáculos, comprometendo fármacos instáveis frente às variações de $\mathrm{pH}$, baixa concentração do fármaco no local de ação considerando curto tempo de residência nos segmentos proximais do TGI. Estas circunstâncias obrigam ao estabelecimento de longos esquemas posológicos, além de elevadas doses, acarretando possíveis reações adversas aos pacientes, e muitas vezes, ineficácia do tratamento (Sasaki, Yamaguti, Cavalcanti, 2002; Mittal et al., 2007; Rajinikanth, Balasubramaniam, Mishra, 2007).

A priori estas circunstâncias podem afetar criticamente produtos gerados a partir da biotecnologia, onde deparamos com fármacos peptídeos, proteínas, vacinas, entre outros. Estes produtos terapêuticos apresentam susceptibilidade tanto à presença das enzimas digestivas, assim como, as flutuações nas faixas de pH, tempo de trânsito, absorção, e distribuição aleatória. Fármacos peptídeos e proteínas são facilmente degradados tanto pela faixa de 
pH estomacal, assim como, pela ação das enzimas proteolíticas disponibilizadas pelo sistema digestório, a instabilidade desses fármacos constitui a razão pela qual eles são administrados pela via parenteral. (Linshu et al., 2003; George, Abraham, 2007).

Este contexto tem sido considerado como verdadeiro desafio às pesquisas farmacêuticas, incentivando inúmeros investimentos na investigação científica na área de tecnologia farmacêutica nas últimas décadas, gerando desenvolvimento de novos sistemas para liberação modificada de fármacos, aliado à perspectiva de uma maior segurança e comodidade aos tratamentos. Desenvolver novos sistemas terapêuticos para a via oral tem sido argumento tecnológico atrativo e justificado pelo número elevado de sistemas disponíveis no mercado farmacêutico, assim como, em fase de desenvolvimento (Sasaki, Yamaguti, Cavalcanti, 2002; Xu et al., 2007; Gabas, Cavalcanti, 2003).

Vários estudos têm sido realizados visando à liberação de fármacos nos segmento distais do TGI, incluindo o íleo e principalmente o colo, onde existe uma fraca disponibilidade das enzimas digestivas, e promissoras perspectivas de ação e/ou absorção de fármacos peptídeos e proteínas naturalmente degradados nos segmentos proximais. O colo apresenta-se como ambiente favorável, considerando faixa de $\mathrm{pH}$ neutro, reduzida atividade enzimática, longo tempo de trânsito, além da presença de uma grande e exclusiva presença de bactérias colônicas as quais secretam enzimas que atacam oligo-polissacarídeos. Certos tipos de oligo-polissacarídeos a exemplo da pectina, quitosana, dextrana, goma guar, galactomana. arabinogalactomana e inulina, têm sido aplicados no desenvolvimento de sistemas enzimaticamente habilitados no controle da liberação de fármacos sítio-alvo-específicos (Ashford et al., 1994; Van Dijk-Wolthuis et al, 1997; Sinha, Kumria, 2001; Codagnone et al., 2004; Cavalcanti et al., 2002; Cavalcanti et al., 2005; Braz, Hechenleitner, Cavalcanti, 2007).

Dentre as alternativas tecnológicas voltadas à pesquisa e desenvolvimento de novos sistemas terapêuticos sítio-alvoespecíficos, o revestimento por compressão destaca-se por apresentar menor tempo de processamento, baixo custo dos equipamentos e passos limitados, baixo requerimento energético e uma técnica que não utiliza solvente. Muitos estudos têm relatado as quantidades e tipos de excipientes utilizados para afetar o perfil de liberação de fármacos, controlando sua biodisponibilidade (Lin, Li, Lin, 2004). Odeku e Fell (2004) avaliaram o processo de revestimento por compressão, utilizando as gomas khaya e albizia como material de revestimento, e a indometacina como fármaco modelo. Turkoglu e Ugurlu (2002) também avaliaram esta técnica a partir de núcleos de ácido 5-amino salicílico revestidos com uma mistura de pectina-HPMC.
Devido às suas vantagens, esta técnica tem motivado várias pesquisas. Estes trabalhos abordam diferentes tecnologias, partindo desde a produção do núcleo até o desenvolvimento propriamente dito do sistema de liberação. Sawada et al. (2004) avaliaram a dependência com o tempo de liberação a partir do material de revestimento, cuja composição consistia de uma mistura de óxido de polietilenopolietileno glicol. Danckwerts e Van der Watt (1995) apresentaram um novo modelo de formato do núcleo revestido, analisando a influência da quantidade de material aplicado no revestimento, a força de compressão, assim como, a espessura, obtendo uma liberação do fármaco de ordem zero. Hariharan e Gupta (2001) propuseram uma nova máquina compressora, na qual o núcleo e o produto revestido são produzidos simultaneamente no mesmo equipamento. Sundy e Danckwerts (2004) apresentaram um comprimido composto por três camadas dotadas de um orifício central, avaliando a aderência entre as camadas, obtiveram resultados de uma liberação próxima de ordem zero, além de mostrar a facilidade de produção em escala industrial.

Dentre os polímeros naturais destaca-se a pectina, polissacarídeo heterogêneo composto de uma cadeia principal linear de unidades repetidas de $(1 \rightarrow 4) \alpha$-D-ácido galacturônico, sendo que parte destas unidades apresenta-se esterificada, isto é, elas possuem um grupo metil (Itoh et al., 2007). As pectinas formam um grupo complexo de polissacarídeos que estão presentes na parede celular de muitas plantas. Industrialmente a extração de pectina ocorre a partir de casca de frutas cítricas (Brandão, Andrade, 1999).

A pectina é uma substância não tóxica, não vulnerável a degradação pelas enzimas digestivas gástricas e/ou intestinais, contribui para aumentar o bolo alimentar, atuando como fibra solúvel, porém é susceptível à degradação pela microflora colônica (Ashford et al., 1993). Devido à sua solubilidade em água, a pectina apresenta limitação na proteção a fármacos durante a sua passagem pelo estômago e intestino delgado (Sinha, Kumria, 2001).

Apesar das expectativas geradas com alguns tipos de pectina, estudos demonstraram que a utilização isolada de alguns polissacarídeos apresenta limitada capacidade no controle da liberação da substância ativa. Desta forma, diversas pesquisas concentram-se em associações entre polissacarídeos e polímeros naturais ou sintéticos (Sasaki, Yamaguti, Cavalcanti, 2002). Estas combinações têm sido propostas na tentativa de combinar diferentes propriedades dos polissacarídeos, em especial, a vulnerabilidade à degradação específica, otimizando as consagradas características dos polímeros sintéticos no controle da liberação de fármacos (Rosina, Baroni, Cavalcanti, 2004).

Dentre estes materiais poliméricos, os derivados da celulose são bastante populares, principalmente a 
hidroxipropilmetil celulose (HPMC). Uma das características mais importantes deste polímero hidrofílico, no que se refere à sua aplicação em sistemas de liberação modificada, é a sua capacidade de intumescimento/relaxamento, a qual exerce efeito pronunciado no controle da cinética de liberação do fármaco incorporado (Lopes et al., 2005).

A HPMC é um polímero hidrofílico linear e os trabalhos realizados demonstram que os seus efeitos se manifestam principalmente pela sua capacidade em formar uma barreira representada pela camada gelatinosa na superfície do comprimido após hidratação. Esta barreira impede a rápida liberação do fármaco, controlando a penetração de água, assim como, a velocidade de liberação da substância ativa. (Lopes et al., 2005).

Turkoglu e Ugurlu (2002) e Ugurlu et al. (2007) demonstraram a partir de ensaios in vitro a versatilidade da mistura pectina-HPMC. Freitas e Cavalcanti (2004) realizaram um estudo dos índices de hidratação de matrizes hidrofílicas contendo goma guar associada ao HPMC em diferentes concentrações, obtiveram resultados que sugerem que a goma guar, quando associada ao HPMC, exerce influência positiva sobre o fenômeno de intumescimento de sistemas matriciais hidrofílicos.

No presente estudo foi desenvolvido sistema sólido oral obtido a partir do processo de revestimento por compressão. Foram aplicados como materiais de recobrimento dois tipos de pectinas de diferentes graus de esterificação (USP-B e 121), associadas a dois tipos de HPMC (Methocel ${ }^{\circledR} \mathrm{K} 100 \mathrm{M}$ e Methocel ${ }^{\circledR} \mathrm{E} 4 \mathrm{M}$ ) de diferentes graus de viscosidade partindo de núcleo obtido pelo processo de compressão direta, tendo como fármaco modelo a teofilina. Foram avaliados os diferentes comportamentos dos sistemas sólidos formados, ou seja, tanto o núcleo quanto o sistema final (núcleo revestido) foram submetidos aos ensaios farmacopeicos convencionais (desintegração do núcleo e peso médio, dureza, friabilidade, espessura tanto para o núcleo como para o comprimido revestido), além da determinação dos índices de hidratação em fluidos de simulação do trato gastrintestinal dos núcleos revestidos.

\section{MATERIAL E MÉTODOS}

\section{Material}

Lactose Super Tab (The Lactose Company of New Zeland Limited - Nova Zelândia), Teofilina Anidra (Henrifarma, São Paulo), Estearato de Magnésio (Henrifarma, São Paulo), Hidroxipropilmetilcelulose (HPMC) - $\left(\right.$ Methoce ${ }^{\circledR}$ K100M Premium CR $80.000-$ $120.000 \mathrm{cP}$ e Methocel E4M Premium CR viscosidade $3.000-5.600 \mathrm{cP}$ ) amostras gentilmente cedidas pela
Colorcon do Brasil (São Paulo), Pectina (USP-B e 121) amostras gentilmente cedidas pela CPKelco (Limeira/SP), máquina de compressão alternativa modelo FABBE, Balança Analítica Mettler Toledo XS 204, Durômetro Erweka $^{\circledR}$ TBH 20, friabilômetro Nova Ética ${ }^{\circledR}$ Mod. 300-1 e Desintegrador ERWEKA ${ }^{\circledR}$ ZT 31.

\section{Métodos}

\section{Preparação dos núcleos de teofilina}

As matérias-primas foram inicialmente pesadas e submetidas à uniformidade da granulometria aplicando tamis de malha $1 \mathrm{~mm}$ e, em seguida, realizou-se a mistura conforme metodologia proposta por Billa e Yuen (2000), para escala piloto. Os núcleos foram obtidos aplicando processo de fabricação por compressão direta em máquina compressora modelo alternativa (FABBE), utilizando punção de $7 \mathrm{~mm}$. A formulação do núcleo consistiu em $100,0 \mathrm{mg}$ de teofilina, 59,6 mg de lactose e 0,4 mg de estearato de magnésio.

\section{Revestimento por compressão dos núcleos}

O processo de revestimento dos núcleos seguiu método proposto por Odeku e Fell (2005). Núcleos de teofilina foram revestidos com diferentes proporções de Pectina e HPMC, sendo usado Pectina 121, cujo grau de esterificação é em média $58 \%$ e Pectina USP tipo B cujo grau de esterificação é em média 72\%, e HPMC E4M (5000cP) e HPMC K100M (120000cP).

Inicialmente foram pesadas $170 \mathrm{mg}$ da mistura envolvendo cada uma das diferentes associações: Pectina / HPMC (proporções: 0:100, 50:50, 60:40, 40:60, 80:20 e 20:80). O material de revestimento (170 mg) foi introduzido na câmara de compressão, contendo jogo de punções de $12 \mathrm{~mm}$. Em seguida, o núcleo de teofilina foi cuidadosamente posicionado no centro da massa, sendo logo em seguida, adicionado o restante $(170 \mathrm{mg})$ do material de revestimento. A compressão do conjunto pós (mistura pectina:HPMC) e núcleos foi realizada utilizando a máquina compressora modelo alternativa. O peso médio dos comprimidos revestidos foi estabelecido em $500 \mathrm{mg}$ e a dureza mantida na faixa de $200 \mathrm{~N}$.

\section{Determinação do peso médio}

Para a determinação do peso médio dos núcleos, foram individualmente pesados 20 comprimidos. A faixa de tolerância preconizada pela Farmacopéia Brasileira 4ª. Ed. (1988) é de 7,5\%. No caso dos comprimidos revestidos, obteve-se o peso médio pesando-se 3 comprimidos, sendo a tolerância (Farmacopéia Brasileira 4a. . Ed., 1988) igual a $5 \%$ (peso superior a $250 \mathrm{mg}$ ). O equipamento utilizado foi 
a Balança Analítica Mettler Toledo XS 204.

\section{Determinação da friabilidade}

A friabilidade foi realizada de acordo com a Farmacopéia Brasileira Ed. 1988. No caso dos núcleos, fez-se $n=20$, e, para os comprimidos revestidos, $n=3$. Os comprimidos foram submetidos à ação de um friabilômetro Nova Ética Mod. 300-1, durante um período de 4 minutos. O resultado foi obtido pela diferença entre as massas inicial e final. Esse valor representa a friabilidade em função da percentagem de pó perdida. O valor preconizado pela Farmacopéia é inferior a 7,5\% do peso estabelecido.

\section{Determinação da espessura}

Os núcleos, assim como, os núcleos revestidos obtidos foram individualmente colocadas no micrômetro Mitutoyo No. 2046F Schok Proof (Japão) para determinação das suas respectivas espessuras.

\section{Determinação da dureza e diâmetro}

Além da espessura, para cada grupo de núcleos não revestidos, e revestidos representando as diferentes composições, foram retiradas amostras para determinação das durezas, usando durômetro Erweka ${ }^{\circledR}$ modelo TBH 20. Sendo $\mathrm{n}=20$ para os núcleos e $\mathrm{n}=3$ para os comprimidos revestidos.

\section{Teste de desintegração dos núcleos}

$O$ teste foi realizado utilizando o desintegrador Erweka ${ }^{\circledR}$ ZT 31. O tempo de desintegração preconizado (Farmacopéia Brasileira 4. Ed. 1988) é de 30 minutos. O teste foi feito $\operatorname{com} n=20$.

\section{Índice de intumescimento (li)}

Os valores do índice de intumescimento foram obtidos a partir do método proposto por Moustafine, Zaharov e Kemenova, 2006. O índice de intumescimento foi determinado a partir de dois diferentes meios, fluido de simulação gástrico $(\mathrm{pH}=1,2)$ e fluido de simulação intestinal $(\mathrm{pH}=6,8)$. Os comprimidos revestidos secos foram pesados (Ps) e, em seguida, foram aprisionados em cesto de desintegrador (Aparatus 2), e imediatamente imersos em fluido de simulação gástrico, permanecendo por um período de 1 hora. Aplicando os mesmos comprimidos, após troca do meio de simulação, estes foram imersos em fluido de simulação intestinal, por um período de 2 horas, mantendo-se a temperatura de $37 \pm 0,5^{\circ} \mathrm{C}$. A cada intervalo de 15 min os comprimidos eram repesados (Pi) e, no final de $180 \mathrm{~min}$ foram fotografados. Os resultados do índice de hidratação foram calculados através da fórmula sugerida por Cavalcanti et al. (2002).

$$
\text { Ii } \%=(\mathrm{Pi}-\mathrm{Ps} / \mathrm{Ps}) \times 100
$$

onde: Pi é o peso do comprimido após o intumescimento e Ps representa o peso do comprimido seco.

\section{Análise Estatística}

As análises estatísticas foram voltadas à determinação do nível de significância existente entre os valores obtidos em todos os ensaios, envolvendo os núcleos revestidos. Estas análises foram realizadas com ajuda do programa GraphPad Prism (versão 2.01, 1996). Os diferentes resultados referentes ao índice de intumescimento foram obtidos em diferentes circunstâncias (meios de simulação do TGI). Foram inicialmente avaliados usando análise de variância (ANOVA). Caso o conjunto desses valores fornecesse parâmetros significativos, realizar-se-á aplicação das médias dos dados obtidos, no teste de múltipla comparação de Tukey, visando comparar o papel das diferentes concentrações dos constituintes. O limite de significância $p=0,05$ foi considerado em ambos os casos (ANOVA e teste de Tukey).

\section{RESULTADOS E DISCUSSÃO}

\section{Características físicas do núcleo de teofilina}

As propriedades físicas dos núcleos de teofilina estão apresentadas na Tabela I. Os núcleos foram produzidos em conformidade com os padrões farmacopéicos.

\section{Características do comprimido revestido}

Os resultados físicos das diferentes composições de revestimento investigadas neste trabalho estão indicados nas Tabelas II e III. Com relação à pectina, por não ser diretamente compressível, não foi possível avaliar a proporção 100:0 (100\% pectina).

TABELA I - Características do núcleo de teofilina \pm desvio padrão, coeficiente de variação (CV)

\begin{tabular}{lccc}
\hline Peso Médio & Dureza $(\mathrm{N})$ & Friabilidade $(\%)$ & Desintegração $(\mathrm{min})$ \\
\hline $160,32(\mathrm{mg}) \pm 1,24$ & $56,5 \pm 4,7$ & 0,9 & 35 \\
$\mathrm{CV}=0,77 \%$ & $\mathrm{CV}=8,32 \%$ & & \\
\hline
\end{tabular}


TABELA II - Características físicas dos comprimidos revestidos com a combinação Pectina 121 - HPMC E4M

\begin{tabular}{lcccccc}
\hline Formulações & $0: 100$ & $20: 80$ & $40: 60$ & $50: 50$ & $60: 40$ & $80: 20$ \\
\hline Peso Médio (mg) & 498,0 & 501,6 & 500,2 & 499,6 & 499,2 & 503,7 \\
Dureza (N) & 195,7 & 210,3 & 206,2 & 195,2 & 204,8 & 202,0 \\
Friabilidade (\%) & 0,20 & 0,58 & 0,98 & 2,77 & 1,05 & 1,32 \\
Espessura (mm) & 0,48 & 0,45 & 0,24 & 0,23 & 0,17 & 0,12 \\
\hline
\end{tabular}

TABELA III - Características físicas dos comprimidos revestidos com a combinação Pectina USP-B - HPMC K100M

\begin{tabular}{lcccccc}
\hline Formulações & $0: 100$ & $20: 80$ & $40: 60$ & $50: 50$ & $60: 40$ & $80: 20$ \\
\hline Peso Médio (mg) & 502,6 & 500,0 & 498,1 & 501,5 & 499,9 & 500,4 \\
Dureza (N) & 208,3 & 203,0 & 200,6 & 208,3 & 199,3 & 209,3 \\
Friabilidade (\%) & 0,08 & 0,07 & 0,15 & 0,09 & 0,18 & 2,40 \\
Espessura (mm) & 0.64 & 0,51 & 0,31 & 0,28 & 0,17 & 0,13 \\
\hline
\end{tabular}

O método aplicado na fabricação a partir da compressão direta, gerou comprimidos revestidos com características físicas adequadas aos padrões farmacopéicos. A pectina quando inserida em maior quantidade gerou comprimidos mais friavéis, menos espessos com exigência de aumento da força de compressão. Conseqüentemente, a maior participação do HPMC conduziu à formação de comprimidos com menor friabilidade.

\section{Índice de Intumescimento (li\%)}

A utilização de diferentes proporções do material de revestimento, além dos diferentes graus de esterificação e viscosidade da pectina e HPMC, respectivamente, teve o intuito de visualizar quais destas formulações teriam a melhor capacidade de intumescer e reter o fármaco de forma adequada. Para tanto, fez-se necessário o uso de condições que reproduzissem as condições do meio fisiológico, obtendo assim resultados in vitro condizentes com o que ocorre no organismo.

A Figura 1 representa um gráfico do índice de intumescimento do revestimento formado por Pectina 121 e HPMC E4M, em diferentes proporções. A proporção $0: 100$ ( $0 \%$ de pectina e $100 \%$ de HPMC) foi usada como controle, assim como na Figura 2, que apresenta os resultados da combinação Pectina USP-B - HPMC K100M.

Na Figura 1, nota-se que a proporção 80:20 intumesce mais rapidamente que as outras proporções, e isto é devido à alta hidrossolubilidade da pectina, o que proporciona grande influxo de água no sistema.

Comparando-se as duas figuras, observa-se que a combinação Pectina 121 - HPMC E4M apresenta maior porcentagem de intumescimento ao início do experimento, devido ao menor grau de esterificação da pectina. Porém, nota-se ao final do experimento um maior índice de hidratação da combinação Pectina USP - HPMC K100M. Isto pode ser explicado pela maior viscosidade do HPMC K100M. No entanto, de acordo com as análises realizadas no programa GraphPad Prism $(2.01,1996)$, não houve uma diferença significativa entre as duas combinações.

A Figura 3 elucida o sistema reservatório formado pelo processo de revestimento por compressão da combinação Pectina/HPMC sobre o núcleo de teofilina, mostrando a eficiência da técnica utilizada, uma vez que o núcleo permaneceu intacto ao final do teste in vitro que simulou as condições do estômago e intestino delgado. A Figura 4 apresenta uma comparação entre os comprimidos seco e intumescido.

\section{CONCLUSÕES}

O método aplicado gerou a formação de comprimidos revestidos obtidos por compressão direta, envolvendo polímero sintético e polissacarídeo (HPMC e pectina) com elevada expectativa sobre o gerenciamento da cinética de liberação de fármacos.

Os resultados obtidos indicam que as combinações propostas aplicando HPMC associado à pectina proporcionaram integridade ao sistema frente às condições de simulação fisiológicas. As diferentes formulações testadas contendo variação da concentração da pectina e/ou HPMC influenciaram a hidratação do sistema. O sistema formado apresenta elevada expectativa no controle da cinética de liberação de fármacos. 


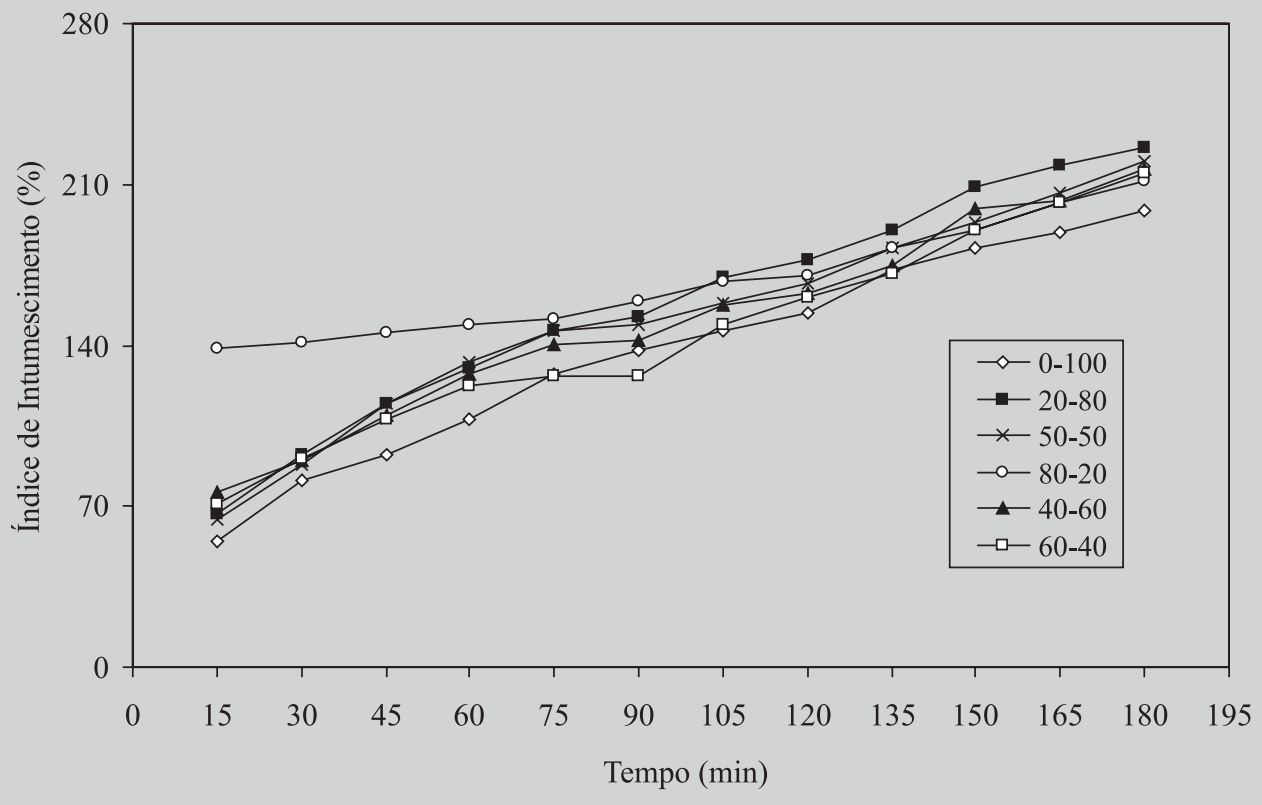

FIGURA 1 - Índice de intumescimento do revestimento formado por Pectina 121 - HPMC E4M em função do tempo. De 0 a $60 \mathrm{~min}(\mathrm{pH} \mathrm{1,2),} \mathrm{e} \mathrm{de} 60$ a $180 \mathrm{~min}(\mathrm{pH} 6,8)$.

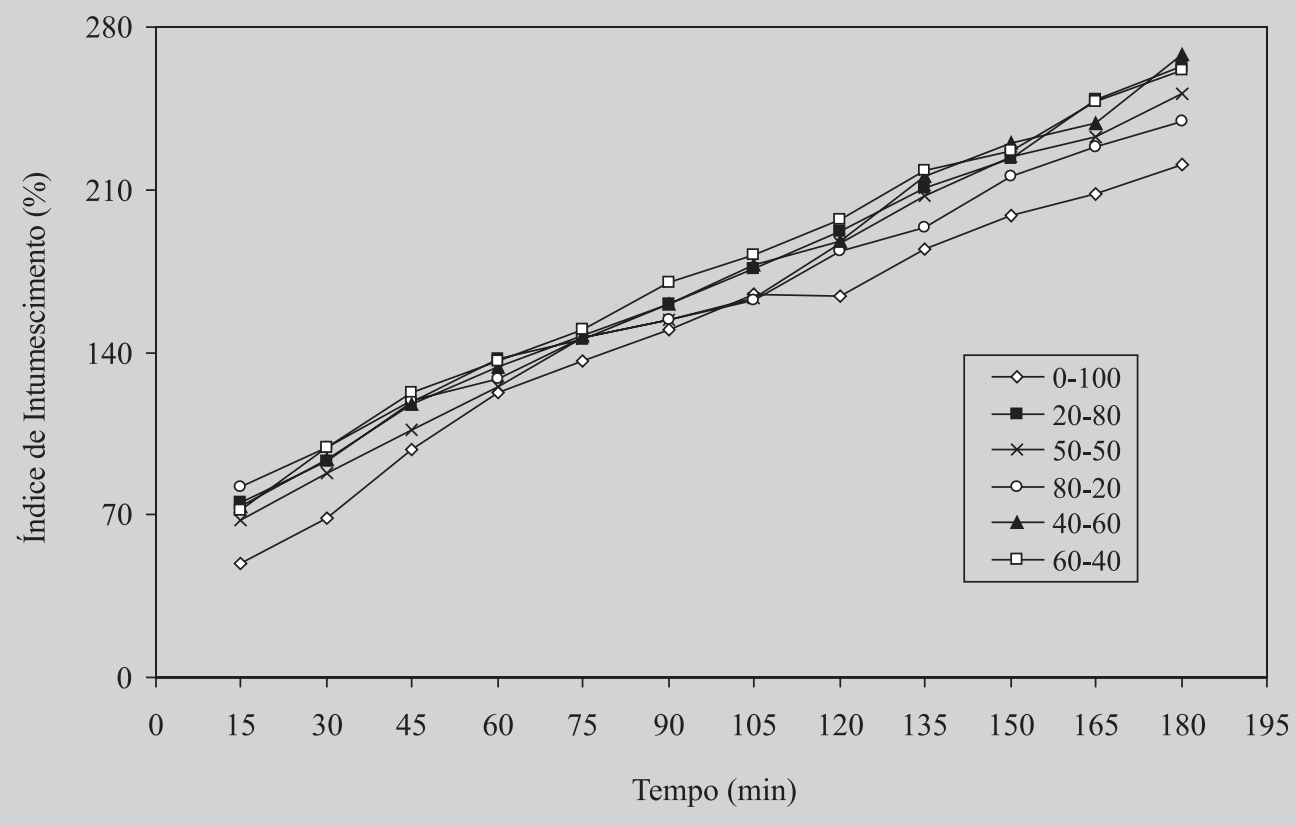

FIGURA 2 - Índice de intumescimento do revestimento formado por pectina USP-B - HPMC K100M em função do tempo. De 0 a 60 min (pH 1,2), e de 60 a 180 min (pH 6,8). 


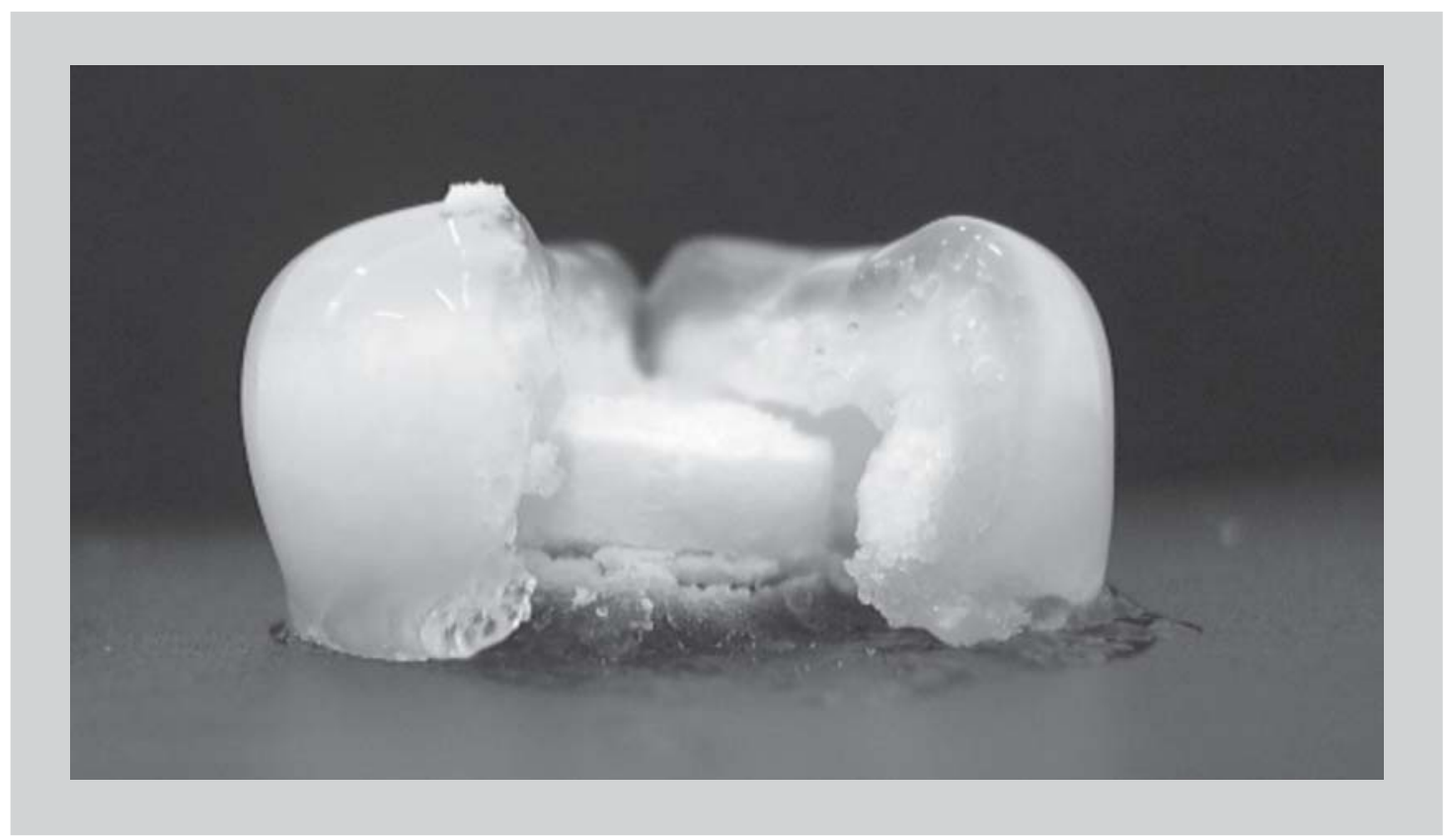

FIGURA 3 - Composição 20:80 (pectina USP/HPMC K100)

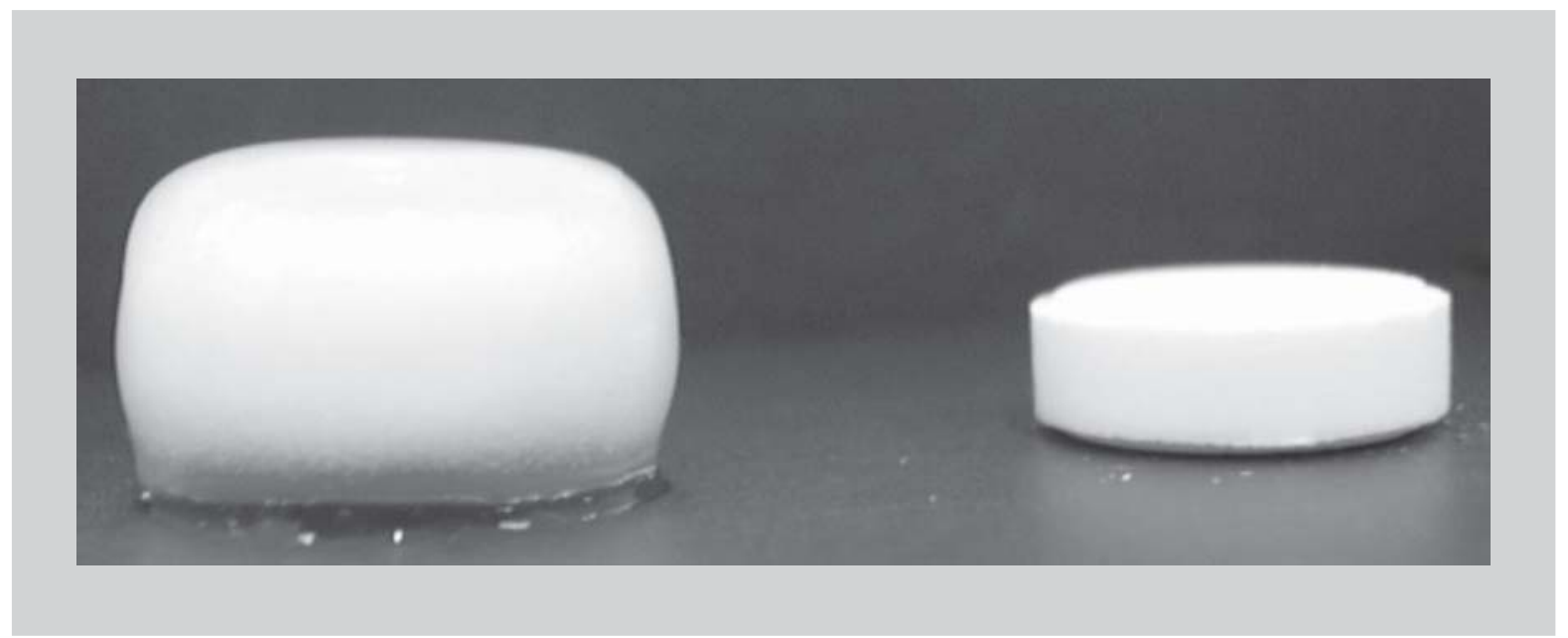

FIGURA 4 - Composição 0:100 (pectina USP/HPMC K100)

\section{AGRADECIMENTOS}

Ao Programa Institucional de Bolsas de Iniciação Científica PIBIC/CNPq-FUNDAÇÃO ARAUCÁRIA UEM, pela concessão de bolsa aos alunos de graduação. Às empresas Colorcon do Brasil e CPKelco pela grande gentileza na cedência das amostras dos produtos Methocel
(K100M e E4M) e Pectina (USP-B e 121), respectivamente. À UPM - Lepemc / UEM pela disponibilidade em ceder seus equipamentos aplicados nos testes físicos. Ao senhor Paulo Sérgio Castanho, técnico do laboratório (DFF-UEM) pela constante gentileza e auxílio no desenvolvimento deste trabalho. Projeto Institucional 3725/ 2005 - PPG/UEM. 


\section{ABSTRACT \\ Evaluation of pectin-hpmc as compression coating. I - A study of the swelling properties of coated tablet}

In this study, core tablets of dry theophylline were compressed coated using the system pectin-HPMC for controlled drug delivery. The methodology applied produced coated tablets with suitable physical characteristics according to Brazil Pharmacopeia $4^{\text {th }}$ edition. The physical properties were evaluated for different ratios (80:20, 60:40, 50:50, 40:60, 20:80, 0:100) of pectin-HPMC, respectively, and the swelling test was carried out in simulated gastric fluid ( $p H 1.2)$ and in simulated intestinal fluid ( $p H$ 6.8). The method applied gave origin to coated tablets with high expectation in the kinetics control of drug release. From the statistics analyses of the results obtained, it was observed that results were not significant between different ratios.

UNITERMS: Tablets/compression coating. Tablets/ evaluation. Theophylline. Pectin. Drugs/release.

\section{REFERÊNCIAS BIBLIOGRÁFICAS}

ASHFORD, M., FELL, J., ATTWOOD, D., SHARMA, H., WOODHEAD, P. An evaluation of pectin as a carrier for drug targeting to the colon. J. Control. Release, Amsterdam, v. 26, p. 213-220, 1993.

ASHFORD, M., FELL, J., ATTWOOD, D., SHARMA, H., WOODHEAD, P. Studies on pectin formulations for colonic drug delivery. J. Control. Release, Amsterdam, v. 30, n.5, p.225-232, 1994.

BILLA, N.; YUEN, K. H. Formulation variables affecting drug release from xantham gum matrices at laboratory scale and pilot scale. AAPS Pharm. Sci. Tech., Arlington, v.1, n.4, p. 1-8, 2000.

BRANDÃO, E. M.; ANDRADE, C. T. Influência de fatores estruturais no processo de gelificação de pectinas de alto grau de metoxilação. Polímeros, São Carlos, v. 9, n. 3, p. 38-44, 1999.

BRAZ, R.; HECHENLEITNER, A. A. W; CAVALCANTI, O. A. Extraction, structural modification and characterization of lotus roots polysaccharides (Nelumbo nucifera Gaertn). Excipient with potential application in modified drug delivery systems. Lat. Am. J. Pharm., v. 26, n. 5, p. 706-710, 2007.
BUNHAK, E. J.; MENDES, E. S.; PEREIRA, N. C.; CAVALCANTI, O. A.. Influência do sulfato de condroitina na formação de filmes isolados de polimetacrilato: avaliação do índice de intumescimento e permeabilidade ao vapor d'água. Quím. Nova, São Paulo, v. 30, n. 2, p. 312-317, 2007.

CAVALCANTI, O. A.; DA SILVA, C. C.; PINEDA, E. A. G.; HECHENLEITNER, A. A. W.. Synthesis and characterization of phosphated crosslinked chondroitin sulfate: Potential ingredient for specific drug delivery. Acta Farm. Bonaerense, La Plata, v. 24.n. 2, p. 234-238, 2005.

CAVALCANTI, O. A.; MOOTER, G. V.; CARAMICOSOARES, I., KINGET, R.. Polysaccharides as excipients for colon-specific coating. Permeability and swelling properties of casted films. Drug Dev. Ind. Pharm., New York, v.28, n.2, p.157-164, 2002.

CODAGNONE, A. F.; HECHENLEITNER, A. A. W.; PINEDA, E. A. G.; CAVALCANTI, O. A.. Phosphated guar gum: Potential ingredient in the development of ethylcellulose isolated films. Acta Farm. Bonaerense, La Plata, v. 23, n. 4, p. 448-452, 2004.

DANKWERTS, M. P.; VAN DER WATT, J. G. The effect of processing variables on the compression properties of controlled release core-in-cup compressed tablets from a new adjustable punch. Int. J. Pharm., Amsterdam, n.123, p.85-94, 1995.

FARMACOPÉIABrasileira. 4. ed. São Paulo: Atheneu, 1988. $6 \mathrm{v}$.

FREITAS,A. B. R.; CAVALCANTI, O. A. Goma Guar como excipiente em matrizes hidrofilicas: avaliação do índice de hidratação, Acta Farm. Bonaerense, La Plata, v. 23, p.334-338, 2004.

GABAS, V. G. S. ; CAVALCANTI, O. A.. Influência da adição da goma arábica em filmes isolados de polímero acrílico. Estudo das propriedades de intumescimento e de permeabilidade. Rev. Bras. Cienc. Farm., São Paulo, v. 39, n. 4, p. 441-448, 2003.

GEORGE, M.; ABRAHAM, T. E. Ph sensitive alginate-guar gum hydrogel for the controlled delivery of protein drugs. Int. J. Pharm., Amsterdam, v. 335, p. 123-129, 2007. 
HARIHARAN, M. ; GUPTA, V. K. A novel compressioncoated tablet dosage form. Pharmac. Technol., New York, p. 14-19, 2001.

ITOH, K.; HIRAYAMA, T.; TAKAHASHI, A.; KUBO, W.; MIYAZAKI, S.; DAIRAKU, M.; TOGASHI, M.; MIKAMI, R.; ATTWOOD, D. In situ gelling pectin formulations for oral drug delivery at high gastric $\mathrm{pH}$. Int. J. Pharm. Amsterdam, v. 335, p. 90-96, 2007.

LINSHU LIU; FISHMAN M. L.; KOST J.; HICKS K. B. Pectin-based systems for colon-specific drug delivery via oral route. Biomaterials, v. 24, p. 3333-3343, 2003.

LIN, S. Y.; LI, M. J.; LIN, K. H. Hydrophilic excipients modulate the time lag of time controlled disintegrating press coated tablets. AAPS PharmSciTech, v.4, n.5, p. 1-5, 2004.

LOPES C. M.; LOBO J. M. S.; COSTA P. Formas farmacêuticas de liberação modificada: polímeros hidrifílicos. Rev. Bras. Ciênc. Farm., São Paulo, v. 41, n. 2, p.143-154, 2005.

MITTAL. G.; SAHANA, D. K.; BHARDWAJ, V.; KUMAR, M. N. V. R.. Estradiol loaded PLGA nanoparticles for oral administration: Effect of polymer molecular weight and copolymer composition on release behavior in vitro and in vivo. J. Control. Release, Amsterdan, v. 119, p. 77-85, 2007.

MOUSTAFINE, R. I.;AHAROV, I. M.; KEMENOVA, V.A. Physicochemical characterization and drug release properties of Eudragit ${ }^{\circledR}$ E PO/Eudragit ${ }^{\circledR}$ L 100-55 interpolyelectrolyte complexes, Eur. J. Pharm. Biopharm., Amsterdan, v. 63, p. 26-36, 2006.

ODEKU, O. A.; FELL, J. T. In vitro evaluation of khaya and albizia gums as coatings for drug targeting to the colon. $J$. Pharm. Pharm., Londres, v. 57, p. 163-168, 2005.

RAJINIKANTH, P. S.; BALASUBRAMANIAM, J.; MISHRA, B. Development and evaluation of a novel floating in situ gelling system of amoxicillin for eradication of Helicobacter pylori. Int. J. Pharm., Amsterdam, v. 335, p. 114-122, 2007.

RIOS, M. Polymers for controlled release: formulation follows function. Pharmac. Technol., New York, v. 29, n. 6, p. 4250, 2005.
ROSINA, C. R., BARONI, S., CAVALCANTI, O. A. Avaliação das propriedades de intumescimento e permeabilidade de filmes isolados de polimetacrilato contendo polissacarídeo da raiz de Lótus (Nelumbo nucifera), Rev. Bras. Cienc. Farm., São Paulo, v. 40, p. 425-431, 2004.

SASAKI, E. H.; YAMAGUTI, E.; CAVALCANTI, O. A. Perspectivas dos polímeros naturais e sintéticos nos sistemas terapêuticos colo-específicos. Rev. Ciênc. Saúde, Maringá, v.2, n.1, p. 49-57, 2002.

SAWADA, T.; KONDO, H.; NAKASHIMA, H.; SAKO, K.; HAYASHI, M.. Time-release compression-coated core tablet containing nifedipine for chronopharmacotherapy. Int. J. Pharm., Amsterdam, v. 280, p. 103-111, 2004.

SINHA, V. R.; KUMRIA, R.. Polysaccharides in colonspecific drug delivery, Int. J. Pharm., Amsterdam, v. 224, p. 19-38, 2001.

SUNDY, E.; DANKWERTS, M. P. A novel compressioncoated doughnut-shaped tablet design for zero-order sustained release. Eur. J. Pharm. Sci., Amsterdam, v.22, p.477-485, 2004.

TURKOGLU, M. ; UGURLU T. In vitro evaluation of PectinHPMC compression coated 5-aminosalicylic acid tablets for colonic delivery. Eur. J. Pharm. Biopharm., Amsterdam, v. 53, p. 65-73, 2002.

UGURLU, T.; TURKOGLU, M.; GURER, U. S.; AKARSU, B. G. Colonic delivery of compression coated nisin tablets using pectin/HPMC polymer mixture. Eur. J. Pharm. Biopharm., Amsterdam, v. 67, p. 202-210, 2007.

VAN DIJK-WOLTHUIS, W. N. E.; VAN STEENBERGEN, M. J.; KETTENES-VAN DEN BOSCH, J. J.; VAN DER KERK-VAN HOOF, A.; HENNINK, W. E. Reaction of dextran with glycidyl methacrylate: an unexpected transesterification. Macromolecules, Washington, v. 30, p. 3411-3413, 1997.

XU, Y.; ZHAN, C.; FAN, L.; WANG, L.; ZHENG, H. Preparation of dual crosslinked alginate-chitosan blend gel beads and in vitro controlled release in oral site-specific drug delivery system. Int. J. Pharm., Amsterdam, v. 336, p. 329-337, 2007.

Recebido para publicação em 13 de junho de 2007. Aceito para publicação em 17 de outubro de 2007. 